\title{
Construction and analysis of an artificial consortium based on the fast-growing cyanobacterium Synechococcus elongatus UTEX 2973 to produce the platform chemical 3- hydroxypropionic acid from $\mathrm{CO} 2$
}

\section{Li Zhang}

Tianjin University

\section{Lei Chen}

Tianjin University

\section{Jinjin Diao}

Tianjin University

\section{Xinyu Song}

Tianjin University

\section{Mengliang Shi}

Tianjin University

Weiwen Zhang ( $\nabla$ wwzhang8@tju.edu.cn )

Tianjin University

\section{Research}

Keywords: Cyanobacteria, Artificial consortium, 3-hydroxypropionic acid, Symbiotic

Posted Date: April 14th, 2020

DOI: https://doi.org/10.21203/rs.2.22486/v2

License: (c) (i) This work is licensed under a Creative Commons Attribution 4.0 International License. Read Full License

Version of Record: A version of this preprint was published at Biotechnology for Biofuels on May 6th, 2020. See the published version at https://doi.org/10.1186/s13068-020-01720-0. 


\section{Abstract}

Background Cyanobacterial carbohydrates, such as sucrose, have been considered as potential renewable feedstock to support the production of fuels and chemicals. However, the separation and purification processes of these carbohydrates will increase the production cost of chemicals. Co-culture fermentation has been proposed as an efficient and economical way to utilize these cyanobacterial carbohydrates. However, studies on the application of co-culture systems to achieve green biosynthesis of platform chemicals are still rare.

Results In this study, we successfully achieved one-step conversion of sucrose derived from cyanobacteria to fine chemicals by constructing a microbial consortium consisting of the fast-growing cyanobacterium Synechococcus elongatus UTEX 2973 and Escherichia coli to sequentially produce sucrose and then the platform chemical 3-hydroxypropionic acid (3-HP) from CO 2 under photoautotrophic growth conditions. First, efforts were made to overexpress the sucrose permeasecoding gene cscB under the strong promoter P cpc560 in S. elongatus UTEX 2973 for efficient sucrose secretion. Second, the sucrose catabolic pathway and malonyl CoA-dependent 3-HP biosynthetic pathway were introduced into E. coli BL21(DE3) for heterologous biosynthesis of 3-HP from sucrose. By optimizing the cultivation temperature from $37^{\circ} \mathrm{C}$ to $30^{\circ} \mathrm{C}$, a stable artificial consortium system was constructed with the capability of producing 3-HP at up to $68.29 \mathrm{mg} / \mathrm{L}$ directly from CO 2 . In addition, cell growth of S. elongatus UTEX 2973 in the consortium was enhanced, probably due to the quick quenching of reactive oxygen species (ROS) in the system by E. coli, which in turn improved the photosynthesis of cyanobacteria.

Conclusion The study demonstrated the feasibility of the one-step conversion of sucrose to fine chemicals using an artificial consortium system. The study also confirmed that heterotrophic bacteria could promote the cell growth of cyanobacteria by relieving oxidative stress in this microbial consortium, which further suggests the potential value of this system for future industrial applications.

\section{Background}

Cyanobacteria are capable of producing organic matter from inorganic carbon $\left(\mathrm{CO}_{2}\right)$ by using solar energy. Due to the challenges associated with global climate change and sustainable energy supply, cyanobacteria have recently attracted significant attention as environmentally friendly and sustainable "microbial cell factories" for the production of biofuels and valuable chemicals directly from $\mathrm{CO}_{2}$ [1]. In addition, cyanobacteria have also been considered a means of producing carbohydrate feedstocks to support industrial fermentative processes [2]. Moreover, it has been reported that several cyanobacterial species are capable of synthesizing and secreting sucrose as an osmolyte under appropriate environmental stimuli, such as osmotic pressure [3], and this production can be sustained over long time periods and at higher levels than that from plant-based feedstocks such as sugarcane and beet [4,5]. As sucrose is an easily fermentable feedstock for many microorganisms [6, 7], significant efforts have been made to improve the production of extracellular sucrose in cyanobacteria [8]. For example, Du et al. 
achieved sucrose productivity at $1.43 \mathrm{mg} / \mathrm{L} / \mathrm{h}$ in wild-type Synechocystis sp. PCC 6803 under $600 \mathrm{mM}$ $\mathrm{NaCl}$ stress in a bioreactor and doubled the productivity to $3.13 \mathrm{mg} / \mathrm{L} / \mathrm{h}$ by co-overexpressing key genes related to sucrose synthesis, namely, sps (s/r0045), spp (s/r0953) and ugp (s/r0207), and deleting the glucosylglycerol phosphate synthase gene ggpS (s//1566) [9]. In another study, Ducat et al. integrated a $\csc B$ gene encoding sucrose permease from Escherichia coli W $[10,11]$ into the Synechococcus elongatus PCC 7942 genome and silenced the carbon competition pathway by knocking out the invertase invA and ADP-glucose pyrophosphorylase glgC to achieve sucrose secretion at a rate of $36.1 \mathrm{mg} / \mathrm{L} / \mathrm{h}$ [12]. Recently, the fast-growing cyanobacterium Synechococcus elongatus UTEX 2973 (hereafter $S$. elongatus UTEX 2973) with a growth rate similar to that of yeast was identified [13], and an extracellular sucrose productivity of $35.5 \mathrm{mg} / \mathrm{L} / \mathrm{h}$ was demonstrated in an engineered S. elongatus UTEX 2973 carrying the sucrose transporter $c s c B$ in a bioreactor experiment [14]. Sucrose productivity was further increased to $79.2 \mathrm{mg} / \mathrm{L} / \mathrm{h}$ through upregulation of $s p s$, which encodes a sucrose-phosphate synthase enzyme, and sucrose synthesis genes in S. elongatus UTEX 2973 [15]. In addition to the high rate of sucrose secretion and growth, this strain exhibits high tolerance to high-temperature $\left(41^{\circ} \mathrm{C}\right)$ and high-light $\left(500 \mu \mathrm{mol}\right.$ photons $\left.\cdot \mathrm{m}^{-2} \cdot \mathrm{s}^{-1}\right)$ conditions, suggesting significant advantages for outdoor cultivation in the future [13]. However, as purification of sucrose from culture supernatant is costly and the system is easily contaminated when sucrose is produced at a large scale [16], alternative ways of utilizing sucrose produced by cyanobacteria need to be developed for potential biotechnological applications.

In nature, microorganisms typically live and interact with other microbes by establishing a stable interchange of substances in complex communities $[17,18]$. Inspired by the commonly found symbiotic relationships of various microbes in nature, studies have been conducted to simulate symbiotic systems by designing artificial routes for the interchange of substances $[19,20]$. Very recently, Ducat et al. constructed a co-culture system with the cyanobacterium S. elongatus PCC 7942 and the heterotrophic bacterium Halomonas boliviensis, in which the growth of $H$. boliviensis was supported by sucrose produced by S. elongatus PCC 7942 [21], and Li et al. constructed a co-culture system consisting of the sucrose secretory cyanobacterium S. elongatus PCC 7942 and three different yeasts to mimic lichen and research the interaction between the autotropic and heterotrophic strains [22]. Although these studies have established new alternatives for the utilization of sucrose derived from cyanobacteria, the use of coculture systems to achieve one-step conversion of sucrose to fine chemicals is still rare [23]. In addition, compared with axenic cultures of cyanobacteria, autotrophic-heterotrophic symbiotic systems have been found to resist contamination effectively and exhibit good robustness in fluctuating environments [24, 25].

3-Hydroxypropionic acid (3-HP, $\mathrm{C}_{3} \mathrm{H}_{6} \mathrm{O}_{3}$ ), as an important platform chemical, is widely used for the production of many chemicals, such as acrylic acid, malonic acid and biodegradable plastic poly-3hydroxypropionic acid, and can also be used as a food additive or preservative[26]. As chemical synthesis of 3-HP causes severe environmental pollution [27], biosynthesis of 3-HP has attracted significant attention recently. Several 3-HP biosynthetic pathways have been reported, and at least four substrates have been used to produce 3-HP, including $\beta$-alanine [28], lactate [29], malonyl-CoA [30] and glycerol [31]. 
Among these pathways, the malonyl-CoA-dependent pathway, which employs acetyl-CoA carboxylase to convert the precursor acetyl-CoA to malonyl-CoA and malonyl-CoA reductase to convert malonyl-CoA to 3HP [32, 33], was reported to have some distinct advantages over other pathways, such as a broad feedstock spectrum, thermodynamic feasibility, and redox neutrality [34]. To date, the malonyl-CoAdependent pathway has been engineered in E. coli [35], Saccharomyces cerevisiae [36], Synechocystis sp. PCC 6803 [37] and S. elongatus PCC 7942 [38] for both heterotrophic and photoautotrophic production of 3-HP [35]. However, until now, no study about the biosynthesis of 3-HP by a co-culture system has been reported.

In this study, we reported the construction of an artificial consortium system consisting of the fastgrowing cyanobacterium S. elongatus UTEX 2973 and an engineered E. coli BL21(DE3) to produce 3-HP under photoautotrophic conditions. In the consortium system, E. coli BL21(DE3) was genetically modified to synthesize 3-HP using sucrose produced by the engineered S. elongatus UTEX 2973 (Fig. 1). With the application of this co-culture system, the final yield of 3-HP was approximately $68.29 \mathrm{mg} / \mathrm{L}$, which is comparable to that obtained in E. coli when only malonyl-CoA reductase was overexpressed [30]. In addition to the relationship where $S$. elongatus provides sucrose as a carbon source for growth and 3-HP production in E. coli, the study also found that increased expression of reactive oxygen species (ROS)quenching genes in E. coli may promote cyanobacterial growth by relieving oxidative stress in the environment.

\section{Results}

\section{Growth of S. elongatus $c s c B^{+} 2973$ and sucrose secretion}

S. elongatus UTEX 2973 was engineered to secrete sucrose by expressing the sucrose permeaseencoding gene $\operatorname{cscB}$ (ECW_m2594) under the strong promoter $P_{c p c 560}$. Sucrose secretion from $S$. elongatus $\operatorname{CscB}^{+} 2973$ is mainly dependent upon the $\mathrm{pH}$ and $\mathrm{NaCl}$ concentration of the medium, and an alkaline environment was previously reported to be beneficial for sucrose secretion from cyanobacterial cells [39]. We used an alkaline environment $(\mathrm{pH} \approx 8.3)$ with $150 \mathrm{mM} \mathrm{NaCl}\left(37^{\circ} \mathrm{C}\right)$ to ensure the production and secretion of sucrose from $S$. elongatus $\csc ^{+} 2973[14,22]$. The sucrose yield and the growth of $S$. elongatus $c s c B^{+} 2973$ in different culture media are compared in Fig. 2. The results showed that no sucrose was produced from S. elongatus $\operatorname{cscB}^{+} 2973$ cells without $\mathrm{NaCl}$ in the culture medium. However, sustainable production and secretion of sucrose could be observed for six days when $150 \mathrm{mM} \mathrm{NaCl}$ was added, and titers of $612.0 \mathrm{mg} / \mathrm{L}$ and $576.5 \mathrm{mg} / \mathrm{L}$ sucrose were achieved when $S$. elongatus $\operatorname{cscB}^{+} 2973$ was grown in BG-11 and CoBG-11, respectively. To maintain the growth of $E$. coli in co-culture medium, the effect of different salt concentrations on cell growth was also examined (Suppl. Fig. S1), and the results showed that $E$. coli was able to grow normally under the tested range of salt concentrations. In this study, a sucrose titer of $576.5-612.0 \mathrm{mg} / \mathrm{L}(4.00-4.25 \mathrm{mg} / \mathrm{L} / \mathrm{h})$ was achieved by $S$. elongatus $\csc B^{+}$ 2973 cells over six days, which is comparable to the levels observed in similar studies conducted previously (Table 2). For example, although no $\mathrm{CO}_{2}$ aeration occurred during $\mathrm{S}$. elongatus cultivation, 
sucrose secretion in this study was still higher than the $2.2 \mathrm{mg} / \mathrm{L} / \mathrm{h}$ value reported in a previous study [22].

\section{Growth of an engineered $E$. coli mutant in co-culture medium}

To ensure that $E$. coli BL21 utilizes sucrose as the sole carbon source, we cloned and expressed the essential genes for sucrose metabolism, namely, $\operatorname{cscB}$ (ECW_m2594), cscK (ECW_m2595) and cscA ( $\left.E C W \_m 2596\right)$, into $E$. coli BL21 to generate an engineered strain, $E$. coli $c s c N$. In addition, to synthesize 3$\mathrm{HP}$, the malonyl-CoA reductase-coding gene $\operatorname{mcr}$ (Caur_2614) was introduced into E. colicscN, resulting in the engineered strain E. coli ABKm. A growth comparison of these two strains is shown in Suppl. Fig. S4. As shown, under the same conditions, the final cell density of $\operatorname{cscN}$ without the $m c r$ gene was slightly increased. In a previous study, an artificial consortium was constructed by inoculating a heterotrophic bacterium into a $S$. elongatus PCC 7942 culture with $\mathrm{OD}_{750}=0.5$ [25]. In our study, the sucrose yield of $S$. elongatus $\csc B^{+} 2973$ was $\sim 200 \mathrm{mg} / \mathrm{L}$ when the cells reached $O D_{750}=0.5$. Therefore, we selected four concentrations, namely, 50,100,150, and $200 \mathrm{mg} / \mathrm{L}$, to examine whether $E$. coli ABKm could be stably maintained in the system using these levels of sucrose as the sole carbon source in M9 and CoBG-11 media (Fig. 3A and 3B). The growth of E. coli ABKm could be detected under 100, 150, and $200 \mathrm{mg} / \mathrm{L}$ sucrose. A previous study showed that the $E$. coli $\triangle c s c R$ strain required a minimal sucrose concentration of $1.2 \mathrm{~g} / \mathrm{L}$ for growth [25], which is much higher than our result for strain ABKm, suggesting that after expression of $\csc A, \csc B$ and $\csc$, the efficiency of sucrose utilization might have improved in strain $A B K m[25,40]$. To demonstrate that this effect was not caused by a strain-specific difference, the sucrose utilization pathway was also engineered into E. coli MG1655 and BW25113, and a similar result was observed (data not shown). Additionally, we also determined the 3-HP yield in strain ABKm with the different concentrations of sucrose mentioned above (i.e. 50 200 mg/L) in CoBG-11, and the results showed that strain ABKm was able to produce 3-HP under all the concentrations except $50 \mathrm{mg} / \mathrm{L}$ sucrose (Fig. 3C).

\section{Establishing a stable artificial consortium to produce 3-HP}

Since the optimal growth temperature for both E. coli and S. elongatus UTEX 2973 is $37^{\circ} \mathrm{C}$, we initially set this as the incubation temperature for the co-culture system. However, the analysis showed that $E$. coli strain ABKm grew poorly after 1 2 days in this system compared with the growth observed in a previous study [25] (Suppl. Fig. S2). According to the data (Fig. 2D), at 37 C, S. elongatus $c s c B^{+} 2973$ cells produce a sufficient amount of sucrose, which prompted us to hypothesize that the rapid cell growth of $E$. coli and 
utilization of sucrose destroy the balance of the two species in this system. To confirm this hypothesis, we determined the rates of sucrose secretion and sucrose utilization in S. elongatus UTEX 2973 and $E$. coli, respectively. The results showed that the sucrose utilization rate of $E$. coli strain ABKm increased gradually with increasing initial sucrose concentration, reaching $\sim 4.20 \mathrm{mg} / \mathrm{L} / \mathrm{h}$ at an initial sucrose concentration of $200 \mathrm{mg} / \mathrm{L}$ with growth at $37^{\circ} \mathrm{C}$ for $48 \mathrm{~h}$ (Fig. 4B). Although the sucrose secretion rate of $S$. elongatus $\csc B^{+}$could reach $\sim 4.11 \mathrm{mg} / \mathrm{L} / \mathrm{h}$, we speculated that with the accumulation of $E$. coli biomass, the sucrose consumption rate of $A B K m$ could be faster than the sucrose secretion rate of $S$. elongatus $\csc B^{+}$at $37^{\circ} \mathrm{C}$. Therefore, the "production-consumption" balance was disrupted, leading to collapse of the consortium. These results led us to adjust the cultivation temperature from $37^{\circ} \mathrm{C}$ to $30^{\circ} \mathrm{C}$, aiming to slow down the consumption of $E$. coli and achieve balanced growth of S. elongatus UTEX 2973 and $E$. coli in the system. The growth of strain $\mathrm{ABKm}$ at $30^{\circ} \mathrm{C}$ was then observed (Fig. 4A), and the sucrose utilization rate of this strain was determined to be $\sim 2.00 \mathrm{mg} / \mathrm{L} / \mathrm{h}$ at $30^{\circ} \mathrm{C}$ at $48 \mathrm{~h}$ (Fig. 4B). Interestingly, there was no significant difference between $37^{\circ} \mathrm{C}$ and $30^{\circ} \mathrm{C}$ in terms of cell growth and sucrose production of the S. elongatus $\csc B^{+} 2973$ strain (Fig. 4C and 4D). As a result, the artificial consortium with S. elongatus $\csc B^{+} 2973$ and E. coli strain ABKm was successfully constructed and could be maintained stably for at least 7 days at $30^{\circ} \mathrm{C}$ (Fig. 4E).

To evaluate the production capacity of the artificial consortium, the 3-HP yield of E. coli strain ABKm was analyzed. As shown in Fig. 5A, 3-HP production reached $\sim 68.29 \mathrm{mg} / \mathrm{L}$ in 7 days. In parallel, we also determined 3-HP production in E. coli strain ABKm under pure culture conditions with continuous supplementation of sucrose according to the calculated sucrose secretion rate of $S$. elongatus $c s c B^{+}$ 2973 (Fig. 5B), and the results showed that the 3-HP yield under pure conditions was at the same level. In addition, we also observed that $S$. elongatus $\csc B^{+} 2973$ cultivated in the consortium grew better than the cells cultivated in pure culture conditions (Fig. 6A), consistent with previous findings [25]. In addition, the results showed that almost no free sucrose could be detected in the co-culture medium, suggesting that sucrose produced by the cyanobacterium was completely consumed by the $E$. coli ABKm strain to support cell growth and accumulate the desired product (Suppl. Fig. S6) [25].

\section{Effect of oxidative stress on cyanobacteria in an artificial consortium system}

ROS are common byproducts of aerobic metabolic processes, such as photoreactions and respiration, in oxygenic photosynthetic organisms [41], and ROS accumulation could cause oxidative damage to cyanobacterial cells. In addition, previous studies have found that organic buffers in culture media may also contribute to the generation of $\mathrm{H}_{2} \mathrm{O}_{2}$ [42]. For example, 1 10 mM 4-(2-hydroxyethyl)1piperazineethanesulfonic acid (HEPES) in culture medium could produce enough $\mathrm{H}_{2} \mathrm{O}_{2}$ to kill Prochlorococcus [43]. Since there was also organic buffer (TES) used to maintain $\mathrm{pH}$ in our study, to 
clarify whether this organic buffer generates $\mathrm{H}_{2} \mathrm{O}_{2}$, we determined the titer of $\mathrm{H}_{2} \mathrm{O}_{2}$ in blank CoBG-11 under the same culture conditions. The results showed that no $\mathrm{H}_{2} \mathrm{O}_{2}$ was detected in blank culture medium, suggesting that the $\mathrm{H}_{2} \mathrm{O}_{2}$ in culture medium was mostly synthesized from living cells. Next, we examined the impact of $E$. colico-cultivation on the $\mathrm{H}_{2} \mathrm{O}_{2}$ level, and the results showed that the $\mathrm{H}_{2} \mathrm{O}_{2}$ content was significantly reduced when the heterotrophic partner of $E$. coli was included in the system

(Fig. 6B), which is consistent with a previous study [22].

To further understand this phenomenon at the molecular level, the expression levels of several $\mathrm{H}_{2} \mathrm{O}_{2}-$ quenching genes in the $E$. coli $\mathrm{ABKm}$ strain under pure and co-culture conditions were comparatively analyzed by qRT-PCR (Suppl. Table. S1). It is well known that E. coli contains three types of catalases: hydroperoxidase I (HPI) (katG), hydroperoxidase II (HPII) (katF), and hydroperoxidase III (HPIII) (katE) [4446]. In addition, the synthesis of HPII often increases markedly when cells enter the stationary phase [47, 48]. The transcriptional expression of these three genes was determined. As shown in Fig. 7, the relative expression levels of katG, katF and katE in the E. coli ABKm strain were dramatically upregulated under co-culture conditions compared with those in CoBG-11 under continuous supplementation with sucrose according to the calculated sucrose secretion rate of $S$. elongatus $c s c B^{+} 2973$, suggesting that $E$. coli might be able to remove ROS when co-cultivated with cyanobacterial partners and thus possibly alleviate the overall oxidative stress in the consortium system.

\section{Discussion}

Photosynthetic cyanobacteria have been considered an important alternative for providing sustainable feedstock, and cyanobacterial carbohydrates have been considered a potential renewable feedstock to support the production of fuels and chemicals. However, since axenic cultures of cyanobacteria are vulnerable to contamination [49] and it is expensive to separate and purify these products from culture medium [50], their application is limited. To address these issues, the use of synthetic consortia of cyanobacteria paired with specific heterotrophic partners has been proposed. The fast-growing $S$. elongatus UTEX 2973 strain reported recently exhibits high tolerance to high-temperature and high-light conditions [13]. In this study, we reported the construction of an artificial co-culture system utilizing $S$. elongatus UTEX 2973 and E. coli $\mathrm{BL} 21$ to produce the heterologous chemical 3-HP directly from $\mathrm{CO}_{2}$. In addition, analysis of the mechanism underlying the co-culture systems also provided a fundamental basis for further optimization of artificial consortium systems via metabolic engineering.

Sucrose is a commonly used carbon source for industrial microbial fermentation [51, 52]. In this study, we transformed the sucrose transporter $\operatorname{cscB}$ into the fast-growing cyanobacterium S. elongatus UTEX 2973 and obtained the sucrose-secreting strain S. elongatus $c s c B^{+} 2973$ with a productivity of $612.0 \mathrm{mg} / \mathrm{L}$ BG11 in six days. To construct an artificial consortium using sucrose as the sole carbon source, the sucrose utilization ability of $E$. coli was also improved. It was previously shown that sucrose utilization could be endowed in the Pseudomonas putida $\csc A B$ strain by introducing the $\csc A$ and $\csc B$ genes from $E$. coli W [23]. To achieve the growth of $E$. coli BL21 in the designed co-culture system, genes related to sucrose 
utilization, namely, $\csc A, \csc B$ and $\csc K$, were introduced [53]. The results showed that this engineering strategy enabled $E$. coli strain ABKm to grow on sucrose as the sole carbon source (Fig. 3). A previous study reported that $E$. coli $\Delta c s c R$ strain required a minimal sucrose concentration of $1.2 \mathrm{~g} / \mathrm{L}$ for growth, which is much higher than the concentration required by $A B K m$ here, suggesting that this engineering strategy significantly improved the efficiency of sucrose utilization [40].

It was previously reported that ROS accumulation in the culture medium severely inhibited axenic growth of cyanobacteria; however, this effect was efficiently alleviated through co-culture with Rhodotorula glutinis [22]. We also observed improved growth of S. elongatus UTEX 2973 in the co-culture system (Fig. 6 A). The $\mathrm{H}_{2} \mathrm{O}_{2}$ content in the consortium system was found to be lower than that in the axenic culture, suggesting that $E$. coli might contribute to the alleviation of ROS stress and thus promote the growth of cyanobacteria in the artificial consortium, which was further confirmed by the upregulation of ROS quenching-related genes in E. coli strain ABKm (Fig. 7). In addition, to confirm our hypothesis, we also tested the influence of $\mathrm{H}_{2} \mathrm{O}_{2}$ on the cell growth of axenic cyanobacterial cultures, and the results showed that the addition of $10 \mu \mathrm{M} \mathrm{H}_{2} \mathrm{O}_{2}$ significantly decreased cell growth (Suppl. Fig. S5). The upregulation of ROS quenching capability could be important, as ROS may deleteriously affect cellular metabolic processes, such as nutrient bioavailability, photosynthesis and carbon flux, in plants [54]. With the decreased $\mathrm{H}_{2} \mathrm{O}_{2}$ content and increased cell growth of $S$. elongatus UTEX 2973, we also evaluated the expression levels of several genes involved in photosynthesis, including the carbon dioxide-concentrating mechanism protein-coding gene $c c m M$ [55], ribulose-1,5-bisphosphate carboxylase/oxygenase (RuBisCO)-coding gene $r b c L$ [56], PSII related subunit-coding genes $c p 43$ and $c p 47$, PSI essential subunits of reaction center-coding genes $p s a A$ and $p s a B$ [57], and chlorophyll a synthesis-related genes chlaA and pcrA [58] (Suppl. Table. S1); as expected, all these genes were upregulated (Suppl. Fig. S3). Several studies have also reported that heterotrophic partners can provide the necessary inorganic carbon by decomposing organic matter and growth factors such as vitamin $\mathrm{B}_{12}$ to cyanobacteria in the natural and artificial consortium $[18,59,60]$, which we will also analyze in future work. Further analysis of the interactions in the consortium could provide the necessary theoretical basis for the potential application of artificial co-culture systems in many areas, such as controlling blooms [61], degradation of pollutants [62-64], and soil remediation [65-67]. For example, Fedeson et al. created an artificial consortium composed of two bacterial species (S. elongatus PCC 7942 and P. putida) that enables the degradation of the industrially produced environmental pollutant 2,4-DNT while simultaneously producing polyhydroxyalkanoates (PHA) bioplastic [68].

Our study also provided some insights into the potential relationships between the two species. The first layer of interaction is that the cyanobacterium provides the carbon source for the $E$. coli strain, and the engineered $E$. coli strain consumes this carbon source to grow and produce fine chemicals. Interestingly, we also found that the cell growth of the cyanobacterium was somewhat improved in the co-culture system. By taking a close look at the changes in the factors involved in determining cell growth, we found that $E$. coli may help quench ROS, which in turn promotes the growth of cyanobacteria, which is 
considered the second layer of interaction between these two species. Collectively, we concluded that the relationship between these two species in the co-culture system is mutualistic.

Although several previous studies have demonstrated promising characteristics of co-culture systems $[21,25]$, our work showed their new application: first, the final product was different. Unlike polyhydroxybutyrate (PHB), 3-HP is a heterologous compound, and our study provides proof of the concept that the production of heterologous fine chemicals can be achieved in co-culture systems by rational design. Second, S. elongatus UTEX 2973 exhibits high tolerance to high-light conditions compared with other model cyanobacterial species, such as S. elongatus PCC 7942, which means that the robustness of our system may be improved. Furthermore, compared with traditional "two-stage" fermentation, this "one-step" strategy also has several unique advantages. Besides the issue of bacterial contamination as the excess sugar in the system is consumed by heterotrophic partners [21], the "twostage" culture strategy typically requires two steps to obtain the chemical production: obtaining the supernatant of the cyanobacterial, and then inoculating the E. coli strain to achieve cell growth and chemical production, which can greatly increase the production cost.

Although the artificial consortium system was successfully established and the desired product was obtained in this study, there remain many aspects that need to be improved in the future, such as the yield of 3-HP. To increase the yield of the target product, on the one hand, we could enhance the supply of the carbon source. In addition to silencing the competing consumption pathway [12], Qiao et al. reported that sucrose yield in S. elongatus PCC 7942 can be enhanced from $6.5 \mathrm{mg} / \mathrm{L} / \mathrm{h}$ to $8.0 \mathrm{mg} / \mathrm{L} / \mathrm{h}$ by overexpressing sucrose-phosphate synthase (sps) and glucose-1-phosphate adenylyltransferase $(g / g C)$ at the same time [69]. The research also suggested that glycogen could serve as a supporting rather than a competitive carbon pool for sucrose synthesis. In addition, Weiss et al. used alginate to encapsulate $S$. elongatus and enhanced the sucrose yield rates $\sim 2$-fold within $66 \mathrm{~h} \mathrm{[21].} \mathrm{On} \mathrm{the} \mathrm{other} \mathrm{hand,} \mathrm{heterotrophic}$ partners also need to be improved. Chelladurai et al. developed a variety of recombinant $E$. coli strains by expressing the heterologous gene $m c r$ and overexpressing the endogenous acetyl-CoA carboxylase and biotinilase-encoding genes $a c c A D B C b$ and nicotinamide nucleotide transhydrogenase-encoding gene pntAB, which converts NADH to NADPH in E. coli. In addition, several deletion mutations in phosphotransacetylase (pta) acetate kinase $(\operatorname{ack} A)$ and lactate dehydrogenase (IdhA) or the aketoglutarate dehydrogenase complex ( $\operatorname{suc} A B$ ) were carried out with the recombinant strains. The final 3HP titer was enhanced approximately 3-fold from 0.71 to $2.14 \mathrm{mM}$ [30]. Moreover, Cheng et al. reported the overexpression of heterogeneous acetyl-CoA carboxylase (from Corynebacterium glutamicum) and codon-optimized $m c r$ in E. coli BL21; three types of modified E. coli strains with different host-vector systems were constructed and investigated, and the results showed that the combination of $E$. coli BL21 and pET28a was the most efficient host-vector system for 3-HP production. The concentration of 3-HP was enhanced from $0.68 \mathrm{~g} / \mathrm{L}$ to $1.80 \mathrm{~g} / \mathrm{L}$ in shake flask cultivation [70]. These studies provide valuable guidance for further metabolic engineering in E. coli.

\section{Conclusion}


With defined composition and controllable functions, synthetic consortia hold great promise for diverse value-added production, bioenergy and environmental applications. In this study, we demonstrated the feasibility of constructing an artificial consortium to achieve the one-step conversion of sucrose to the platform chemical 3-HP directly from $\mathrm{CO}_{2}$. With the application of this co-culture system, the final production of 3-HP was approximately $68.29 \mathrm{mg} / \mathrm{L}$, which is comparable to that in $E$. coli when only malonyl-CoA reductase was overexpressed. Meanwhile, this study also confirmed that in this microbial consortium, heterotrophic bacteria could promote the cell growth of cyanobacteria by relieving oxidative stress, which further demonstrates the potential value of this system for the green biosynthesis of chemicals in the future.

\section{Material And Method}

\section{Strains, plasmid construction and culture conditions}

S. elongatus UTEX 2973 and E. coli BL21(DE3) were engineered and applied to the construction of the artificial consortium system. The essential genes for sucrose metabolism, namely, the permease-coding gene $\csc B$, invertase-coding gene $\csc A$ and fructokinase-coding gene $c s c K$, were derived from $E$. coli W [53], while the malonyl-CoA reductase gene mor was from Chloroflexus aurantiacus [71]. The super-strong promoter $P_{c p c 560}$ was used to direct gene expression in S. elongatus UTEX 2973 [72]. All plasmids were prepared in E. coli DH5a. The sucrose hydrolysis system was integrated into pET-30a in E. coli with kanamycin resistance, and mcr was integrated into pACYC184 under the constitutive promoter $P_{J 23100}$ in E. coli with spectinomycin resistance.

S. elongatus UTEX 2973 and the resulting engineered strains were cultivated in BG-11 medium (pH 7.5) under a light intensity of approximately $100 \mu \mathrm{mol}$ photons $\mathrm{m}^{-2} \mathrm{~s}^{-1}$ in an illuminating shaking incubator (HNYC-202T, Honour, Tianjin, China) at $130 \mathrm{rpm}$ and $37^{\circ} \mathrm{C}$ or on BG-11 agar plates in an incubator (SPX250B-G, Boxun, Shanghai, China) [73]. To maintain the stable phenotype of sucrose secretion, appropriate antibiotics were added when necessary. E. coli strains were grown on LB medium or agar plates with appropriate antibiotics added to maintain plasmids at $37^{\circ} \mathrm{C}$ in a shaking incubator (HNY-100B, Honour, Tianjin, China) at $200 \mathrm{rpm}$ or in an incubator, respectively. All strains used in this study are listed in Table 1.

\section{Conjugation of S. elongatus UTEX 2973}

Constructs were delivered into S. elongatus UTEX 2973 through conjugation [74]. E. coli HB101 harboring pRL443 and pRL623 (named "helper") and E. coliDH5a harboring the plasmid with the target gene were cultivated overnight and then transferred separately into fresh liquid LB medium with the appropriate antibiotics at a 1:50 ratio. When both strains grew to exponential phase $\left(\mathrm{OD}_{600}=0.3 \sim 0.5\right), 10 \mathrm{~mL}$ of the 
cells of each strain was collected by centrifugation and washed with fresh LB medium three times to remove all the antibiotics. Then, $0.1 \mathrm{~mL}$ of fresh LB was used to resuspend each strain, and the cells were mixed together and incubated at $37^{\circ} \mathrm{C}$ for $30 \mathrm{~min}$. During this time, $10 \mathrm{~mL}$ of $\mathrm{S}$. elongatus UTEX 2973 cells at exponential phase $\left(\mathrm{OD}_{750} \approx 1\right)$ was collected by centrifugation and resuspended in $0.2 \mathrm{~mL}$ of fresh BG-11 medium. S. elongatus UTEX 2973 cells were mixed with the $E$. coli mixture mentioned above and incubated at $37^{\circ} \mathrm{C}$ under light for $30 \mathrm{~min}$. Then, the mixture was spread on BG-11 agar plates, which were then covered by sterile cellulose filters $(0.45 \mu \mathrm{m}$ pore size). The plates were incubated under light at an intensity of approximately $100 \mu \mathrm{mol}$ photons $\mathrm{m}^{-2} \mathrm{~s}^{-1}$ for $24 \mathrm{~h}$, and then, the cellulose filters were transferred onto new BG-11 agar plates with appropriate antibiotics [75].

\section{Construction of the artificial consortium system}

Co-culture medium (named CoBG-11) was designed based on BG-11 medium and optimized for E. coli growth by supplementing with $150 \mathrm{mM} \mathrm{NaCl}, 4 \mathrm{mM} \mathrm{NH}_{4} \mathrm{Cl}$ and $3 \mathrm{~g} / \mathrm{L}$ 2-[[1,3-dihydroxy-2-(hydroxymethyl) propan-2-yl] amino] ethanesulfonic acid (TES). The $\mathrm{pH}$ value was adjusted with $\mathrm{NaOH}$ to 8.3. $\mathrm{NaCl}$ and $\mathrm{NH}_{4} \mathrm{Cl}$ were used to maintain the cell survival of $E$. coli, and $\mathrm{NaCl}$ was used as a stress inducer for sucrose accumulation in S. elongatus UTEX 2973.

Before the two strains were cultivated together, S. elongatus UTEX 2973 was propagated in BG- 11 at $37^{\circ} \mathrm{C}$ with appropriate antibiotics to the exponential phase $\left(\mathrm{OD}_{750} \approx 1.0\right)$, collected by centrifugation, inoculated into $25 \mathrm{~mL}$ of CoBG- 11 medium and grown at $30^{\circ} \mathrm{C}$ for $48 \mathrm{~h}$ to an $O D_{750}$ of 0.5 . E. coli was incubated in CoBG-11 with $1 \mathrm{~g} / \mathrm{L}$ sucrose for $48 \mathrm{~h}$ and then collected by centrifugation, resuspended in deionized water and inoculated into the $25-\mathrm{mL} S$. elongatus culture described above at an initial $\mathrm{OD}_{600}$ of 0.01 .

\section{Quantification of cyanobacteria and E. coli}

For pure cultures of S. elongatus UTEX 2973 and E. coli, cell density was measured at $\mathrm{OD}_{750}$ and $\mathrm{OD}_{600}$, respectively, using a UV-1750 spectrophotometer (Shimadzu, Kyoto, Japan). For co-culture, serial dilutions were made, and solid LB agar plates were used to determine $E$. coli viability and cell number by counting colony-forming units (CFU) after $24 \mathrm{~h}$ of incubation at $37^{\circ} \mathrm{C}$. The cell number of $S$. elongatus UTEX 2973 was determined by a hemocytometer under a microscope (BX43, Olympus, Shinjuku, Tokyo, Japan). 


\section{Determination of extracellular sucrose content}

Supernatants of pure S. elongatus UTEX 2973 cultures were collected and analyzed for sucrose content via a colorimetric glucose-sucrose assay (Megazyme, Ireland) that employs high-purity glucose oxidase, peroxidase and $\beta$-fructosidase (invertase). At pH 4.6, sucrose is hydrolysed by invertase to D-glucose and $D$-fructose, and then, the free D-glucose content is determined by conversion to a red-colored quinoneimine dye compound through the action of glucose oxidase and peroxidase at $\mathrm{pH} 7.4$ and employing $p$-hydroxybenzoic acid and 4-aminoantipyrine. Measurements were conducted at $510 \mathrm{~nm}$.

\section{Quantification of 3-HP}

The 3-HP concentration was quantified according to a previously described method [37]. A 3-HP standard of analytical purity was purchased from Tokyo Chemical Industry (Tokyo, Japan). The supernatant containing 3-HP was collected from the co-culture medium by centrifuging at 12,000 rpm for $2 \mathrm{~min}$ at room temperature (Eppendorf 5430R, Hamburg, Germany) and used for 3-HP analysis. Sample derivatization was carried out according to the two-stage technique described previously [76]. GC-MS analysis was conducted on a GC-MS system-GC 7890 coupled to an MSD 5975 (Agilent Technologies, Inc., Santa Clara, CA) equipped with an HP-5MS capillary column (30 mm×250 mm id).

\section{Quantitative real-time RT-PCR analysis}

Approximately $4 \times 10^{6}$ pure or co-cultured S. elongatus UTEX 2973 cells were collected by centrifugation at $12,000 \mathrm{rpm}$ and $4^{\circ} \mathrm{C}$ for $1 \mathrm{~min}$. The supernatant was removed, and the cells were used for RNA extraction and RT-qPCR analysis using methods described previously [77]. The relative abundance of different mRNA molecules could be estimated using $2^{-\triangle \triangle C T}$ [78].

\section{Analysis of $\mathrm{H}_{2} \mathrm{O}_{2}$ concentration}

The $\mathrm{H}_{2} \mathrm{O}_{2}$ content in the supernatant was analyzed using the $\mathrm{H}_{2} \mathrm{O}_{2}$ Quantitative Assay Kit (Sangon Biotech, Shanghai, China). In the reaction, $\mathrm{Fe}^{2+}$ is oxidized to $\mathrm{Fe}^{3+}$ by $\mathrm{H}_{2} \mathrm{O}_{2}$ when the $\mathrm{pH}$ is less than 7.0, and then, the generated $\mathrm{Fe}^{3+}$ combines with dye molecules to form a claret-colored $\mathrm{Fe}^{3+}$-dye complex with a maximum absorption wavelength of $560 \mathrm{~nm}$ or $595 \mathrm{~nm}$, and the absorption value is directly proportional to the concentration of $\mathrm{H}_{2} \mathrm{O}_{2}$ in cells. 


\section{Abbreviations}

GC-MS, gas chromatography-mass spectrometry; WT, wild type; 3-HP, 3-hydroxypropionic acid; F6P, fructose 6-phosphate; PHA, polyhydroxyalkanoates; PHB, polyhydroxybutyrate; ROS, reactive oxygen species.

\section{Declarations}

Ethical approval and consent to participate

Not applicable.

Consent for publication

All authors have agreed to the publication of this manuscript.

Availability of supporting data

All data generated or analyzed during this study are included in this published article and its additional files.

Competing interests

The authors declare no competing financial interests.

Funding

This research was supported by grants from the National Key Research and Development Program of China (grant no. 2019YFA0904600, 2018YFA0903000 and 2018YFA0903600), the National Natural Science Foundation of China (no. 91751102, 31901016, 31770035, 31770100, 31972931, 21621004, 31370115 and 31470217), and Tianjin Synthetic Biotechnology Innovation Capacity Improvement Project (no. TSBICIP-KJGG-007).

Authors' contributions 
XYS and WZ designed the research; LZ performed the major experiments and wrote the draft manuscript; JJD and XYS helped with some of the experiments; MLS helped with the GC-MS analysis; and LZ, LC, $\mathrm{XYS}$ and WZ analyzed the data and drafted and revised the manuscript. All authors read and approved the final manuscript.

Acknowledgements

None

\section{References}

1. Quintana N, Van der Kooy F, Van de Rhee MD, Voshol GP, Verpoorte R: Renewable energy from Cyanobacteria: energy production optimization by metabolic pathway engineering. Appl Microbiol Biotechnol 2011, 91:471-490.

2. Hays SG, Ducat DC: Engineering cyanobacteria as photosynthetic feedstock factories. Photosynthesis Res 2015, 123:285-295.

3. Hagemann M: Molecular biology of cyanobacterial salt acclimation. FEMS Microbiol Rev 2011, 35:87-123.

4. Niederholtmeyer $\mathrm{H}$, Wolfstädter BT, Savage DF, Silver PA, Way JC: Engineering cyanobacteria to synthesize and export hydrophilic products. Appl Environ Microbiol 2010, 76:3462-3466.

5. Han Y, Watson M: Production of microbial levan from sucrose, sugarcane juice and beet molasses. $J$ Ind Microbio/ 1992, 9:257-260.

6. Reid SJ, Abratt VR: Sucrose utilisation in bacteria: genetic organisation and regulation. App/ Microbiol Biotechnol 2005, 67:312-321.

7. Feng J, Gu Y, Quan Y, Gao W, Dang Y, Cao M, Lu X, Wang Y, Song C, Wang S: Construction of energyconserving sucrose utilization pathways for improving poly-y-glutamic acid production in Bacillus amyloliquefaciens. Microbial cell factories 2017, 16:98.

8. Kirsch F, Klähn S, Hagemann M: Salt-regulated accumulation of the compatible solutes sucrose and glucosylglycerol in cyanobacteria and its biotechnological potential. Front Microbio/ 2019, 10:2139.

9. Du W, Liang F, Duan Y, Tan X, Lu X: Exploring the photosynthetic production capacity of sucrose by cyanobacteria. Metab Eng 2013, 19:17-25.

10. Sahintoth M, Frillingos S, Lengeler JW, Kaback HR: Active transport by the cscB permease in Escherichia coli K-12. Biochem Biophys Res Commun 1995, 208:1116-1123.

11. Vadyvaloo V, Smirnova IN, Kasho VN, Kaback HR: Conservation of residues involved in sugar/ $\mathrm{H}^{+}$ symport by the sucrose permease of Escherichia coli relative to lactose permease. J Mol Biol 2006, 358:1051-1059. 
12. Ducat DC, Avelar-Rivas JA, Way JC, Silver PA: Rerouting carbon flux to enhance photosynthetic productivity. App/ Environ Microbio/ 2012, 78:2660-2668.

13. Yu J, Liberton M, Cliften PF, Head RD, Jacobs JM, Smith RD, Koppenaal DW, Brand JJ, Pakrasi HB: Synechococcus elongatus UTEX 2973, a fast growing cyanobacterial chassis for biosynthesis using light and $\mathrm{CO}_{2}$. Sci Rep 2015, 5:8132.

14. Song K, Tan X, Liang Y, Lu X: The potential of Synechococcus elongatus UTEX 2973 for sugar feedstock production. Appl Microbiol Biotechnol 2016, 100:7865-7875.

15. Lin P-C, Zhang F, Pakrasi HB: Enhanced production of sucrose in the fast-growing cyanobacterium Synechococcus elongatus UTEX 2973. Sci Rep 2020, 10:1-8.

16. Chisti Y: Constraints to commercialization of algal fuels. J Biotechnol 2013, 167:201-214.

17. Beliaev AS, Romine MF, Serres M, Bernstein HC, Linggi BE, Markillie LM, Isern NG, Chrisler WB, Kucek LA, Hill EA: Inference of interactions in cyanobacterial-heterotrophic co-cultures via transcriptome sequencing. The ISME journal 2014, 8:2243.

18. Christie-Oleza JA, Sousoni D, Lloyd M, Armengaud J, Scanlan DJ: Nutrient recycling facilitates longterm stability of marine microbial phototroph-heterotroph interactions. Nature microbiology 2017, 2:17100.

19. Fredrickson JK: Ecological communities by design. Science 2015, 348:1425-1427.

20. Brenner K, You L, Arnold FH: Engineering microbial consortia: a new frontier in synthetic biology. Trends Biotechnol 2008, 26:483-489.

21. Weiss TL, Young EJ, Ducat DC: A synthetic, light-driven consortium of cyanobacteria and heterotrophic bacteria enables stable polyhydroxybutyrate production. Metab Eng 2017, 44:236-245.

22. Li T, Li C-T, Butler K, Hays SG, Guarnieri MT, Oyler GA, Betenbaugh MJ: Mimicking lichens: incorporation of yeast strains together with sucrose-secreting cyanobacteria improves survival, growth, ROS removal, and lipid production in a stable mutualistic co-culture production platform. Biotechnology for biofuels 2017, 10:55.

23. Löwe H, Hobmeier K, Moos M, Kremling A, Pflüger-Grau K: Photoautotrophic production of polyhydroxyalkanoates in a synthetic mixed culture of Synechococcus elongatus $\csc B$ and Pseudomonas putida cscAB. Biotechnology for biofuels 2017, 10:190.

24. Rippka R, Deruelles J, Waterbury JB, Herdman M, Stanier RY: Generic assignments, strain histories and properties of pure cultures of cyanobacteria. Microbiology 1979, 111:1-61.

25. Hays SG, Yan LL, Silver PA, Ducat DC: Synthetic photosynthetic consortia define interactions leading to robustness and photoproduction. J Biol Eng 2017, 11:4.

26. Gokarn RR, Selifonova OV, Jessen HJ, Gort SJ, Selmer T, Buckel W: 3-Hydroxypropionic acid and other organic compounds. Google Patents; 2007.

27. Jiang X, Meng X, Xian M: Biosynthetic pathways for 3-hydroxypropionic acid production. App/ Microbiol Biotechnol 2009, 82:995-1003. 
28. Borodina I, Kildegaard KR, Jensen NB, Blicher TH, Maury J, Sherstyk S, Schneider K, Lamosa P, Herrgård MJ, Rosenstand I: Establishing a synthetic pathway for high-level production of 3hydroxypropionic acid in Saccharomyces cerevisiae via $\beta$-alanine. Metab Eng 2015, 27:57-64.

29. Henry CS, Broadbelt LJ, Hatzimanikatis V: Discovery and analysis of novel metabolic pathways for the biosynthesis of industrial chemicals: 3-hydroxypropanoate. Biotechnol Bioeng 2010, 106:462473.

30. Rathnasingh C, Raj SM, Lee Y, Catherine C, Ashok S, Park S: Production of 3-hydroxypropionic acid via malonyl-CoA pathway using recombinant Escherichia coli strains. J Biotechnol 2012, 157:633640.

31. Raj SM, Rathnasingh C, Jo J-E, Park S: Production of 3-hydroxypropionic acid from glycerol by a novel recombinant Escherichia coli BL21 strain. Process Biochem 2008, 43:1440-1446.

32. Zhang W, Song X: Synthetic Biology of Cyanobacteria. Springer; 2018.

33. Hügler M, Menendez $\mathrm{C}$, Schägger $\mathrm{H}$, Fuchs $\mathrm{G}$ : Malonyl-coenzyme A reductase from Chloroflexus aurantiacus, a key enzyme of the 3-hydroxypropionate cycle for autotrophic $\mathrm{CO}_{2}$ fixation. $\mathrm{J}$ Bacteriol 2002, 184:2404-2410.

34. Liu C, Ding Y, Xian M, Liu M, Liu H, Ma Q, Zhao G: Malonyl-CoA pathway: a promising route for 3hydroxypropionate biosynthesis. Crit Rev Biotechnol 2017, 37:933-941.

35. Liu C, Wang Q, Xian M, Ding Y, Zhao G: Dissection of malonyl-coenzyme A reductase of Chloroflexus aurantiacus results in enzyme activity improvement. PloS one 2013, 8:e75554.

36. Chen Y, Bao J, Kim I-K, Siewers V, Nielsen J: Coupled incremental precursor and co-factor supply improves 3-hydroxypropionic acid production in Saccharomyces cerevisiae Metab Eng 2014, 22:104109.

37. Wang Y, Sun T, Gao X, Shi M, Wu L, Chen L, Zhang W: Biosynthesis of platform chemical 3hydroxypropionic acid (3-HP) directly from $\mathrm{CO}_{2}$ in cyanobacterium Synechocystis sp. PCC 6803. Metab Eng 2016, 34:60-70.

38. Lan El, Chuang DS, Shen CR, Lee AM, Ro SY, Liao JC: Metabolic engineering of cyanobacteria for photosynthetic 3-hydroxypropionic acid production from $\mathrm{CO}_{2}$ using Synechococcus elongatus PCC 7942. Metab Eng 2015, 31:163-170.

39. Chi X, Zhang S, Mao S, Luan G, Luo Q, Lü X: Cyanobacteria based photosynthetic production of sucrose: development and prospect. Chinese journal of biotechnology 2019, 35:1411-1423.

40. Sabri S, Nielsen LK, Vickers CE: Molecular control of sucrose utilization in Escherichia coli W, an efficient sucrose-utilizing strain. App/ Environ Microbio/ 2013, 79:478-487.

41. Polerecky L, Bachar A, Schoon R, Grinstein M, Jørgensen BB, De Beer D, Jonkers HM: Contribution of Chloroflexus respiration to oxygen cycling in a hypersaline microbial mat from Lake Chiprana, Spain. Environ Microbiol 2007, 9:2007-2024.

42. Ferreira CM, Pinto IS, Soares EV, Soares HM: (Un) suitability of the use of pH buffers in biological, biochemical and environmental studies and their interaction with metal ions-a review. RSC 
Advances 2015, 5:30989-31003.

43. Jeffrey Morris J, Zinser ER: Continuous hydrogen peroxide production by organic buffers in phytoplankton culture media. J Phycol 2013, 49:1223-1228.

44. Loewen PC: Isolation of catalasedeficient Escherichia coli mutants and genetic mapping of katE, a locus that affects catalase activity. J Bacterio/ 1984, 157:622-626.

45. Loewen P, Triggs B: Genetic mapping of katF, a locus that with katE affects the synthesis of a second catalase species in Escherichia coli. J Bacteriol 1984, 160:668-675.

46. Loewen PC, Triggs BL, George CS, Hrabarchuk BE: Genetic mapping of katG, a locus that affects synthesis of the bifunctional catalase-peroxidase hydroperoxidase I in Escherichia coli. $J$ Bacteriol 1985, 162:661-667.

47. Hassan HM, Fridovich I: Regulation of the synthesis of catalase and peroxidase in Escherichia coli. $J$ Biol Chem 1978, 253:6445-6420.

48. Loewen PC, Switala J, Triggs-Raine BL: Catalases HPI and HPII in Escherichia coli are induced independently. Arch Biochem Biophys 1985, 243:144-149.

49. Gavrilescu M, Chisti Y: Biotechnology-a sustainable alternative for chemical industry. Biotechnol Adv 2005, 23:471-499.

50. Ziolkowska JR: Prospective technologies, feedstocks and market innovations for ethanol and biodiesel production in the US. Biotechnology Reports 2014, 4:94-98.

51. Wittmann $\mathrm{C}$, Kiefer $\mathrm{P}$, Zelder $\mathrm{O}$ : Metabolic fluxes in Corynebacterium glutamicum during lysine production with sucrose as carbon source. App/ Environ Microbiol 2004, 70:7277-7287.

52. Lee IY, Seo WT, Kim GJ, Kim MK, Park C, Park YH: Production of curdlan using sucrose or sugar cane molasses by two-step fed-batch cultivation of Agrobacterium species. J Ind Microbiol Biotechnol 1997, 18:255-259.

53. Li F, Ma J, Wu M, Ji Y, Chen W, Ren X, Jiang M: Succinic acid production from sucrose and sugarcane molasses by metabolically engineered Escherichia coli. Chinese journal of biotechnology 2015, 31:534-541.

54. Hasanuzzaman M, Bhuyan M, Anee TI, Parvin K, Nahar K, Mahmud JA, Fujita M: Regulation of ascorbate-glutathione pathway in mitigating oxidative damage in plants under abiotic stress. Antioxidants 2019, 8:384.

55. Kerfeld CA, Melnicki MR: Assembly, function and evolution of cyanobacterial carboxysomes. Curr Opin Plant Biol 2016, 31:66-75.

56. Liang F, Lindberg P, Lindblad P: Engineering photoautotrophic carbon fixation for enhanced growth and productivity. Sustainable Energy \& Fuels 2018, 2:2583-2600.

57. Armond P, Staehelin L, Arntzen C: Spatial relationship of photosystem I, photosystem II, and the lightharvesting complex in chloroplast membranes. The Journal of cell biology 1977, 73:400-418.

58. Papageorgiou GC, Tsimilli-Michael M, Stamatakis K: The fast and slow kinetics of chlorophyll a fluorescence induction in plants, algae and cyanobacteria: a viewpoint. Photosynthesis Res 2007, 
94:275-290.

59. Bernstein HC, McClure RS, Thiel V, Sadler NC, Kim Y-M, Chrisler WB, Hill EA, Bryant DA, Romine MF, Jansson JK: Indirect interspecies regulation: transcriptional and physiological responses of a cyanobacterium to heterotrophic partnership. MSystems 2017, 2:e00181-00116.

60. Ren M, Zhang G, Ye Z, Qiao Z, Xie M, Lin Y, Li T, Zhao J: Metagenomic analysis reveals potential interactions in an artificial coculture. Amb Express 2017, 7:193.

61. Zhu L, Zancarini A, Louati I, De Cesare S, Duval C, Tambosco K, Bernard C, Debroas D, Song L, Leloup $\mathrm{J}$ : Bacterial communities associated with four cyanobacterial genera display structural and functional differences: evidence from an experimental approach. Front Microbio/ 2016, 7:1662.

62. Liu J, Wu Y, Wu C, Muylaert K, Vyverman W, Yu H-Q, Muñoz R, Rittmann B: Advanced nutrient removal from surface water by a consortium of attached microalgae and bacteria: a review. Bioresour Technol 2017, 241:1127-1137.

63. Yang Z, Pei H, Hou Q, Jiang L, Zhang L, Nie C: Algal biofilm-assisted microbial fuel cell to enhance domestic wastewater treatment: nutrient, organics removal and bioenergy production. Chemical Engineering Journal 2018, 332:277-285.

64. Alcántara C, Domínguez JM, García D, Blanco S, Pérez R, García-Encina PA, Muñoz R: Evaluation of wastewater treatment in a novel anoxic-aerobic algal-bacterial photobioreactor with biomass recycling through carbon and nitrogen mass balances. Bioresour Technol 2015, 191:173-186.

65. Abou-Shady A: Reclaiming salt-affected soils using electro-remediation technology: PCPSS evaluation. Electrochim Acta 2016, 190:511-520.

66. Dahlawi S, Naeem A, Rengel Z, Naidu R: Biochar application for the remediation of salt-affected soils: Challenges and opportunities. Sci Total Environ 2018, 625:320-335.

67. Nain L, Rana A, Joshi M, Jadhav SD, Kumar D, Shivay Y, Paul S, Prasanna R: Evaluation of synergistic effects of bacterial and cyanobacterial strains as biofertilizers for wheat. Plant and soil 2010, 331:217-230.

68. Fedeson DT, Saake P, Calero P, Nikel PI, Ducat DC: Biotransformation of 2, 4-dinitrotoluene in a phototrophic co-culture of engineered Synechococcus elongatus and Pseudomonas putida. bioRxiv 2018:404988.

69. Qiao C, Duan Y, Zhang M, Hagemann M, Luo Q, Lu X: Effects of reduced and enhanced glycogen pools on salt-induced sucrose production in a sucrose-secreting strain of Synechococcus elongatus PCC 7942. Appl Environ Microbiol 2018, 84:e02023-02017.

70. Cheng Z, Jiang J, Wu H, Li Z, Ye Q: Enhanced production of 3-hydroxypropionic acid from glucose via malonyl-CoA pathway by engineered Escherichia coli. Bioresour Technol 2016, 200:897-904.

71. d Mattozzi M, Ziesack M, Voges MJ, Silver PA, Way JC: Expression of the sub-pathways of the Chloroflexus aurantiacus 3-hydroxypropionate carbon fixation bicycle in E. coli. Toward horizontal transfer of autotrophic growth. Metab Eng 2013, 16:130-139.

72. Wang B, Eckert C, Maness P-C, Yu J: A genetic toolbox for modulating the expression of heterologous genes in the cyanobacterium Synechocystis sp. PCC 6803. ACS synthetic biology 2017, 7:276-286. 
73. Stanier R, Kunisawa R, Mandel M, Cohen-Bazire G: Purification and properties of unicellular bluegreen algae (order Chroococcales). Bacteriol Rev 1971, 35:171.

74. Elhai J, Wolk CP: Conjugal transfer of DNA to cyanobacteria. In Methods in enzymology. Volume 167: Elsevier; 1988: 747-754

75. Li S, Sun T, Xu C, Chen L, Zhang W: Development and optimization of genetic toolboxes for a fastgrowing cyanobacterium Synechococcus elongatus UTEX 2973. Metab Eng 2018, 48:163-174.

76. Roessner U, Luedemann A, Brust D, Fiehn O, Linke T, Willmitzer L, Fernie AR: Metabolic profiling allows comprehensive phenotyping of genetically or environmentally modified plant systems. The Plant Cell 2001, 13:11-29.

77. Gao W, Zhang W, Meldrum DR: RT-qPCR based quantitative analysis of gene expression in single bacterial cells. J Microbiol Methods 2011, 85:221-227.

78. Livak KJ, Schmittgen TD: Analysis of relative gene expression data using real-time quantitative PCR and the $2^{-\triangle \Delta C T}$ method. Methods 2001, 25:402-408.

\section{Additional File Information}

Additional file 1:

Suppl. Fig. S10Growth of E. coli ABKm under different concentrations of $\mathrm{NaCl}$ in $\mathrm{CoBG}-11$ medium at $37^{\circ} \mathrm{C}$.

Suppl. Fig. S2: Growth in the artificial consortium system at $37^{\circ} \mathrm{C}$. S. elongatus $\csc B^{+}$(green square) and E. coli ABKm (gray diamond).

Suppl. Fig. S3: Expression level analysis of genes involved in photosynthesis. Gene expression analysis of $c c m M, r b c L, c p 43, c p 47, p s a B, p s a A$. chlaA and pcrA in S. elongatus $c s c B^{+}$. The error bars represent the calculated standard deviation of the measurements of three biological replicates.

Suppl. Fig. S4: Growth of E. coli cscN and ABKm. A), B), C) Cultivated in M9 medium; D), E), F) cultivated in CoBG-11 medium.

Suppl. Fig. S5: Growth of the cyanobacterium S. elongatus $\csc B^{+}$with $\mathrm{H}_{2} \mathrm{O}_{2}$ added.

Suppl. Fig. S6: Consumption of sucrose in the co-culture system.

Additional file 2:

Table S1. Related genes and primers used in this study.

\section{Tables}


Table 1. Plasmids and strains used in this study.

\begin{tabular}{|c|c|c|}
\hline Plasmids/Strains & Genotype & Source \\
\hline \multicolumn{3}{|l|}{ E. coli strains } \\
\hline DH5 $\alpha$ & $\begin{array}{l}\mathrm{F}^{-} \text {endA1 glnV44 thi- } \\
1 \text { recA1 relA1 gyrA96 deoR nup purB20 } 980 \mathrm{dlacZ \Delta M} 15 \Delta(\operatorname{lac} Z Y A- \\
\arg F) \mathrm{U} 169, \mathrm{hsdR} 17\left(r_{K}^{-} m_{K}^{+}\right), \lambda^{-}\end{array}$ & $\begin{array}{l}\text { TransGen } \\
\text { Biotech }\end{array}$ \\
\hline HB101 & $\begin{array}{l}\text { sup E44, } \Delta(\operatorname{mcr} C-m r r), \operatorname{rec} A 13 \text {, ara-14, proA2, lacY1, galK2, rpsL20, xyl-5, } \\
\text { mtl-1, leuB6, thi-1 }\end{array}$ & $\begin{array}{l}\text { Takara } \\
\text { Bio }\end{array}$ \\
\hline BL21(DE3) & $\mathrm{F}^{-}$ompT gal dcm lon $h s d S_{B}\left(r_{B}^{-} m_{B}^{-}\right) \lambda(\mathrm{DE} 3)$ & $\begin{array}{l}\text { TransGen } \\
\text { Biotech }\end{array}$ \\
\hline $\operatorname{cscN}$ & BL21(DE3)/pET30a & \\
\hline $\mathrm{ABKm}$ & BL21(DE3)/pACYC184/pET30a & This study \\
\hline \multicolumn{3}{|l|}{ Plasmids } \\
\hline pACYC184 & $\mathrm{P}_{J 23100-\mathrm{mcr}-\mathrm{T}_{7} ; s p e^{R}}$ & This study \\
\hline pET30a & $\csc \mathrm{A}, \csc \mathrm{B}, \csc \mathrm{K} ; \mathrm{kan}^{R}$ & This study \\
\hline pJA & 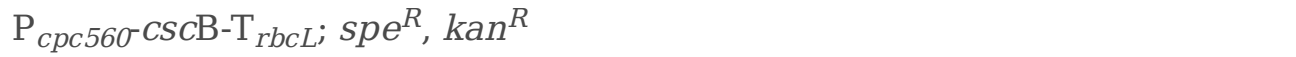 & This study \\
\hline \multicolumn{3}{|l|}{$\begin{array}{l}\text { Cyanobacteria } \\
\text { strains }\end{array}$} \\
\hline WT & wild type Synechococcus elongatus UTEX 2973 & \\
\hline $\operatorname{CsCB} B^{+} 2973$ & WT/pJA & This study \\
\hline
\end{tabular}

Table 2. Sucrose yield in different cyanobacteria strains under co-culture conditions. 


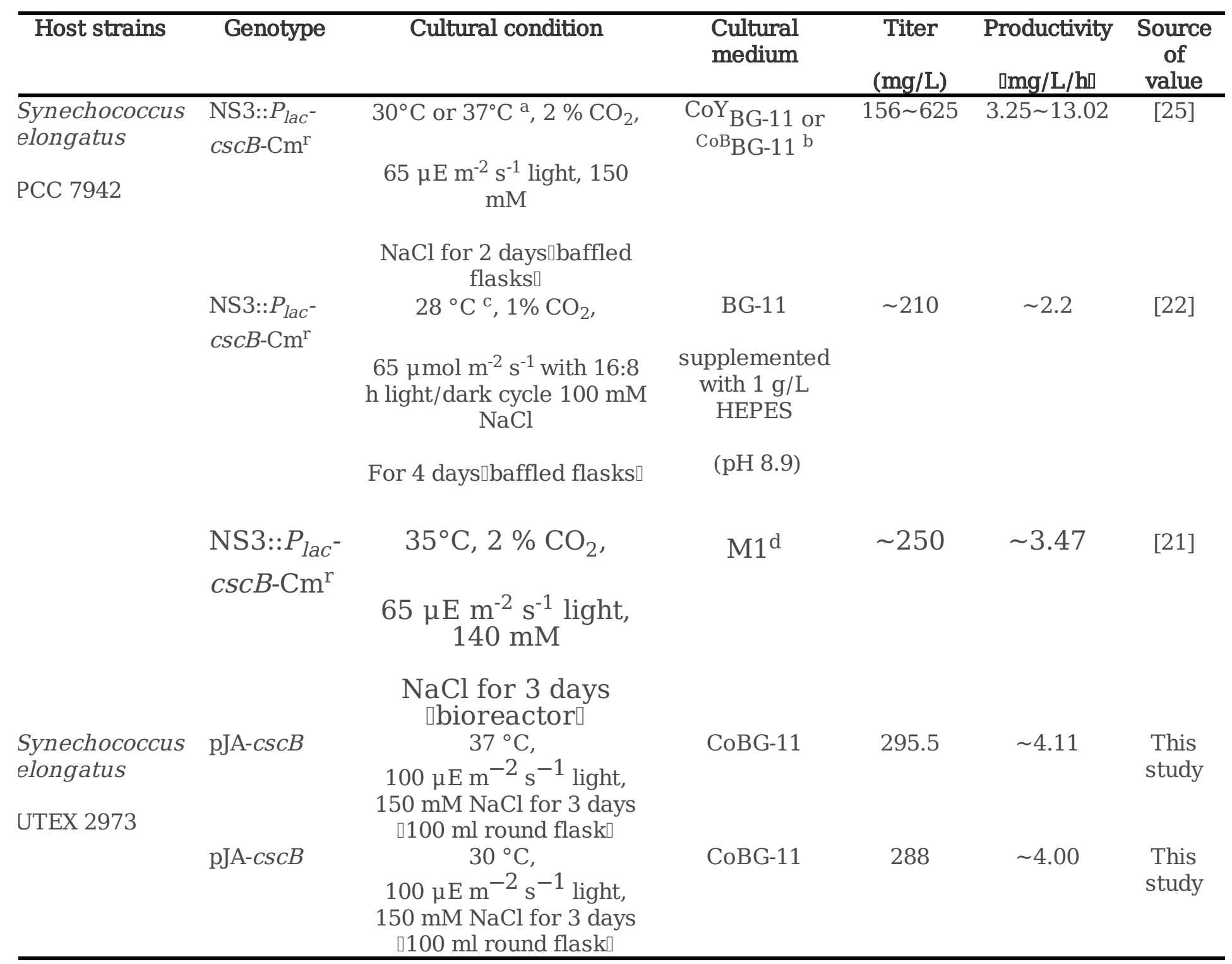

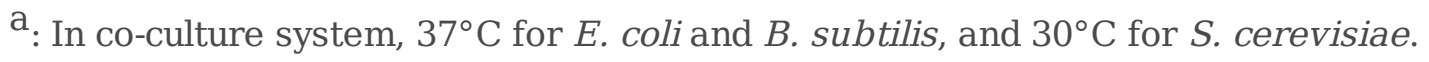

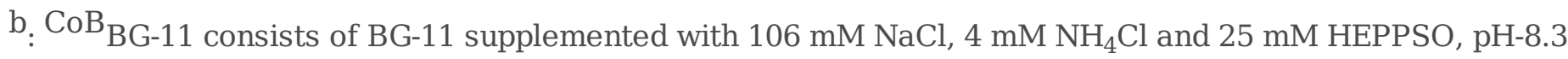

$\mathrm{KOH} .{ }^{\mathrm{CoY}}{ }_{\mathrm{BG}-11}$ consists of BG-11 supplemented with $0.36 \mathrm{~g} / \mathrm{L}$ Yeast Nitrogen Base without amino acids (Sigma Aldrich), $106 \mathrm{mM} \mathrm{NaCl}, 25 \mathrm{mM}$ HEPPSO, $\mathrm{pH}$ 8.3-KOH and $1 \mathrm{mM} \mathrm{KPO}_{3}$.

C: In co-culture system, $28^{\circ} \mathrm{C}$ for three yeast strains.

d: M1 consists BG-11 medium was additionally supplemented with $15 \mathrm{mM} \mathrm{NaNO}_{3}, 4.5 \mathrm{mM} \mathrm{K}_{2} \mathrm{HPO}_{4}$ (phosphate buffering), $1.5 \mathrm{mM} \mathrm{MgSO}_{4}, 5 \mathrm{mM} \mathrm{Na}_{2} \mathrm{SO}_{4}, 30 \mu \mathrm{M} \mathrm{FeCl}_{3}, 30 \mu \mathrm{M} \mathrm{Na}_{2} \mathrm{MoO}_{4}$, and 1x additional trace metals before the addition of $\mathrm{KOH}$ to $\mathrm{pH} 8.3$.

\section{Figures}


$\mathrm{CO}_{2}$

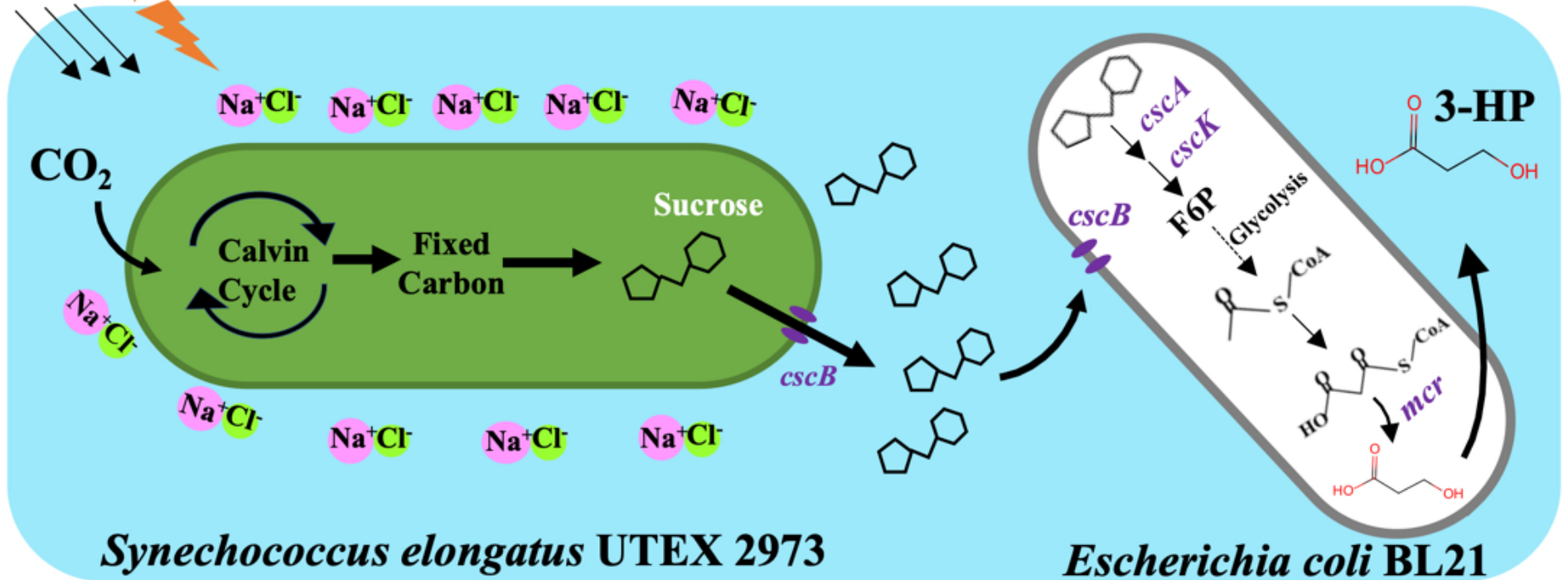

Figure 1

Schematic diagram of the artificial consortium system. The engineered S. elongatus UTEX 2973 secreted sucrose under osmotic stress to support E. coli growth and synthesis of 3-HP under photoautotrophic growth conditions. (F6P, fructose 6-phosphate; 3-HP, 3-hydroxypropionic acid)
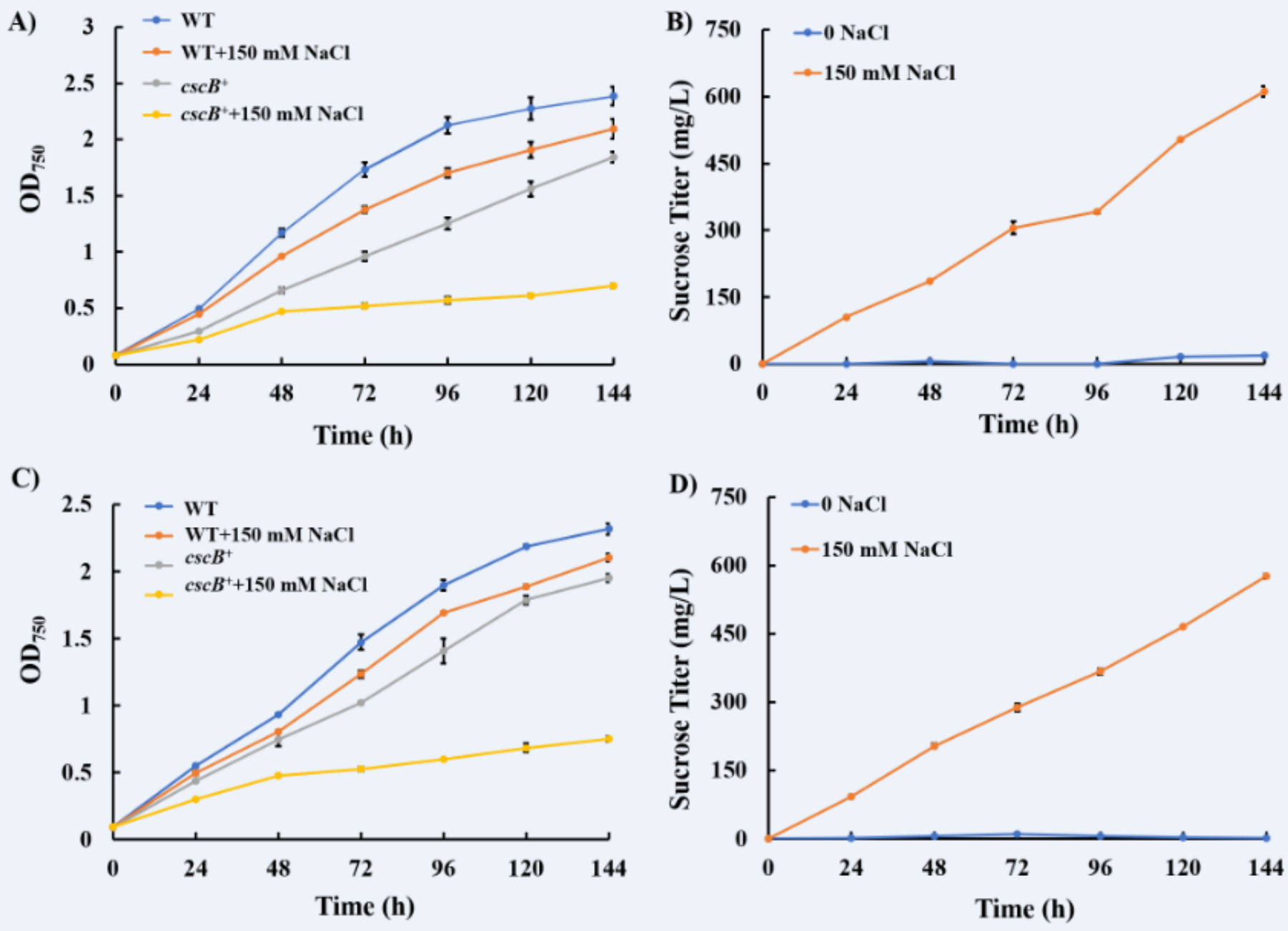
Figure 2

Effects of $\mathrm{NaCl}$ on the growth and sucrose yield of $\mathrm{S}$. elongatus $\operatorname{cscB}+$. Growth of strain $\mathrm{S}$. elongatus cscB+ in BG-11 medium (A) and CoBG-11 medium (C). Sucrose yield of strain S. elongatus cscB+ in BG11 medium (B) and CoBG-11 medium (D). The error bars represent the calculated standard deviation of the measurements of three biological replicates.
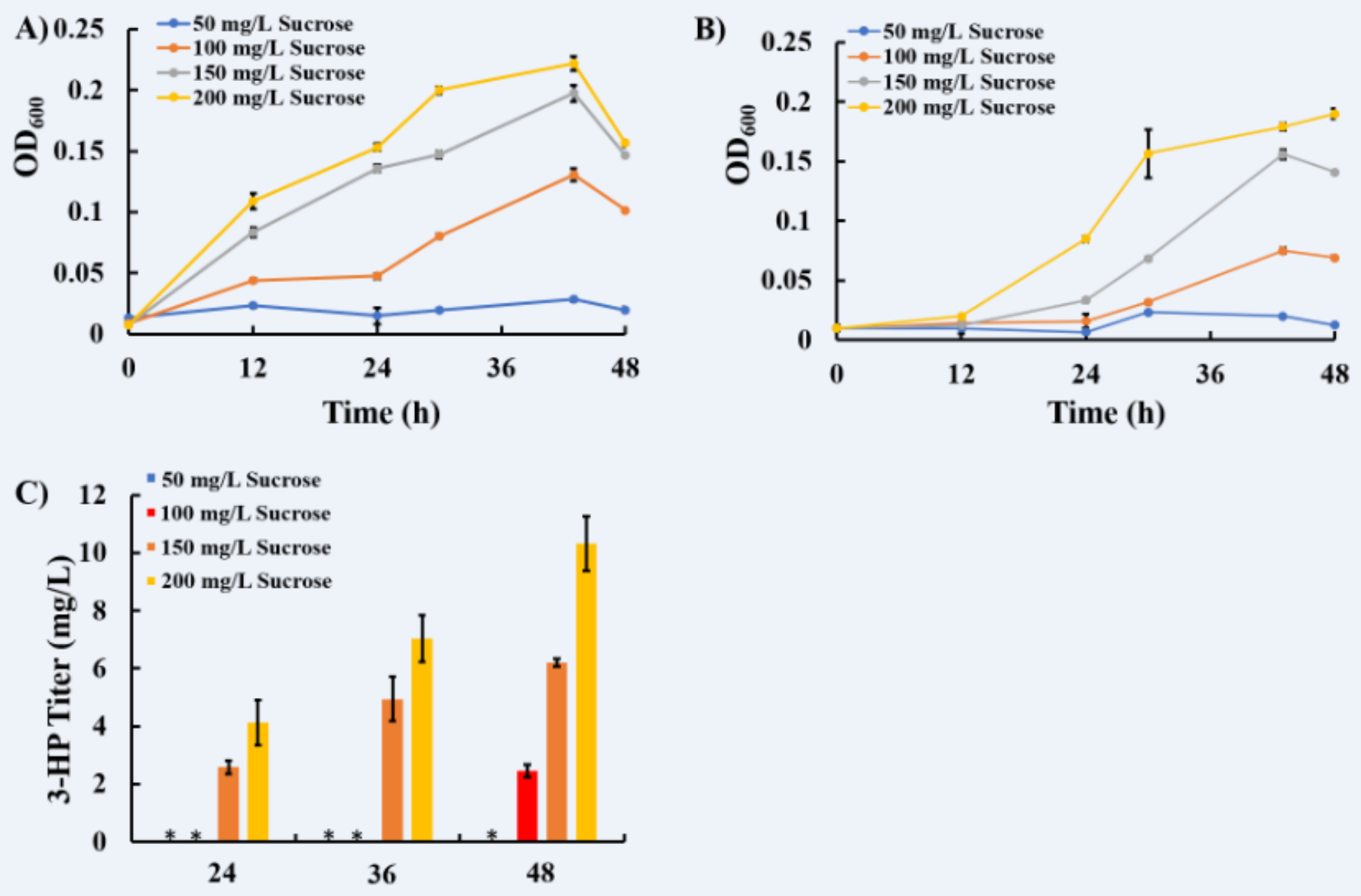

Time (h)

Figure 3

Growth and 3-HP production of pure E. coli ABKm culture under different sucrose concentrations. (A) Cultivated in M9 medium; (B) cultivated in CoBG-11 medium. The error bars represent the calculated standard deviation of the measurements of three biological replicates. (C) 3-HP production in the E. coli ABKm strain under different initial sucrose concentrations in co-culture medium (" $\star$ " indicates that no detectable amount was observed). The error bars represent the calculated standard deviation of the measurements of three biological replicates. 

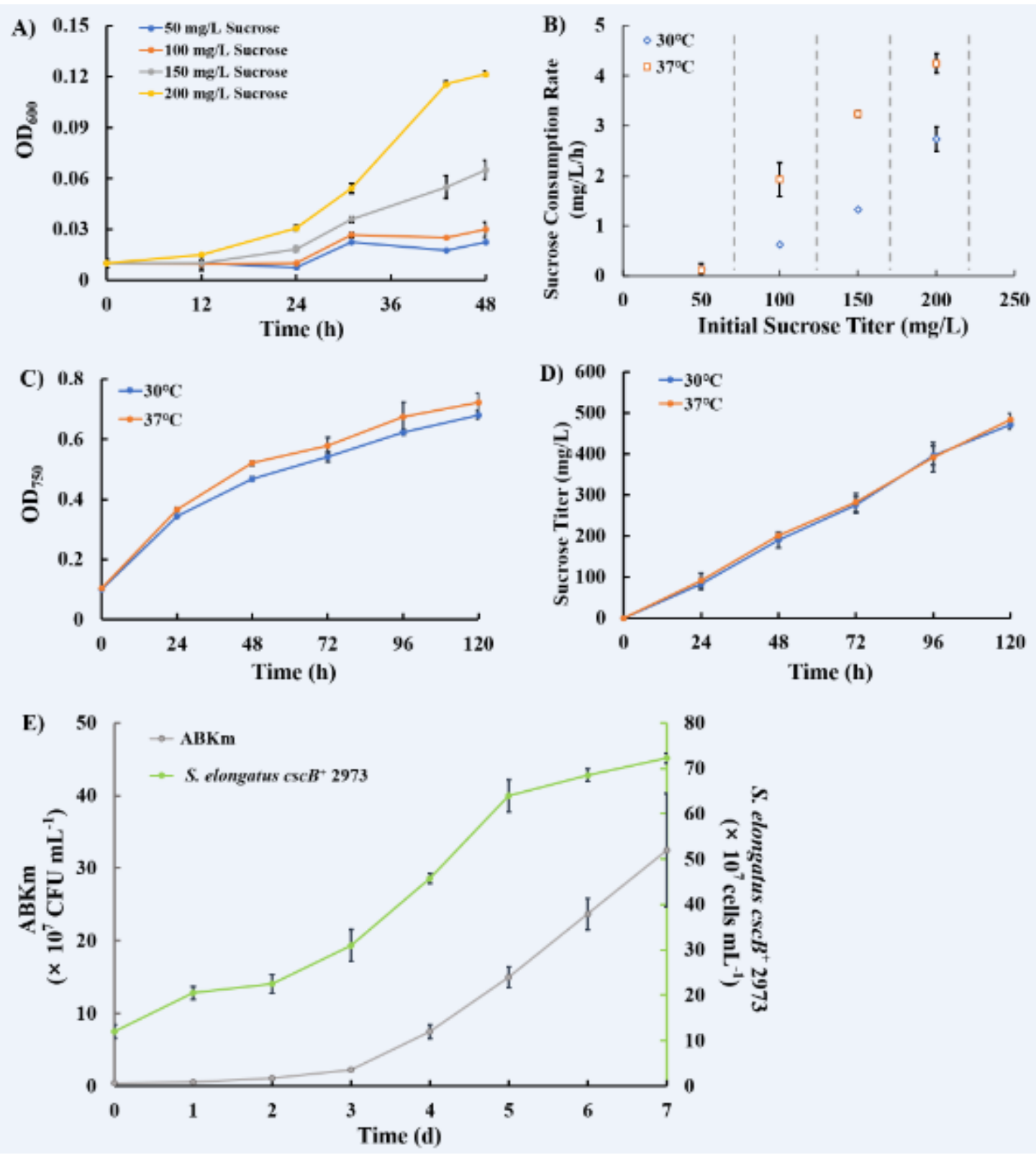

Figure 4

Construction of an artificial consortium system with engineered S. elongatus UTEX 2973 and E. coli BL21. (A) Growth of pure ABKm culture in CoBG-11 medium with different concentrations of sucrose at $30^{\circ} \mathrm{C}$. (B) Sucrose utilization rate of pure ABKm culture. (C) Growth of pure S. elongatus UTEX 2973 culture at $30^{\circ} \mathrm{C}$ and $37^{\circ} \mathrm{C}$. (D) Sucrose yield of engineered S. elongatus $\operatorname{cscB}+$. (E) Growth of two strains in the artificial consortium system at $30^{\circ} \mathrm{C}$. The error bars represent the calculated standard deviation of the measurements of three biological replicates. 


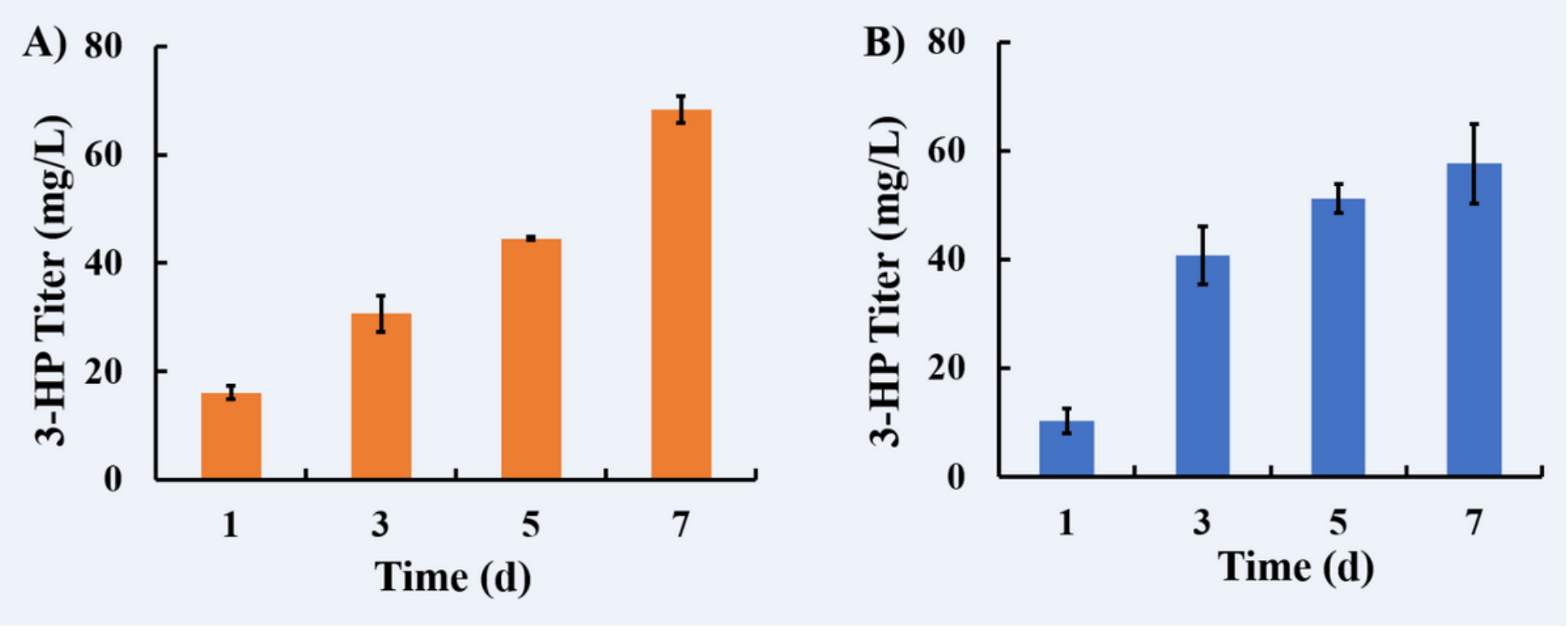

\section{Figure 5}

3-HP yield under different conditions. (A) 3-HP yield in an artificial consortium system. (B) 3-HP production in strain $A B K m$ under continuous supplementation with sucrose according to the calculated sucrose secretion rate of $\mathrm{S}$. elongatus $\mathrm{cscB}+$. The error bars represent the calculated standard deviation of the measurements of three biological replicates. 

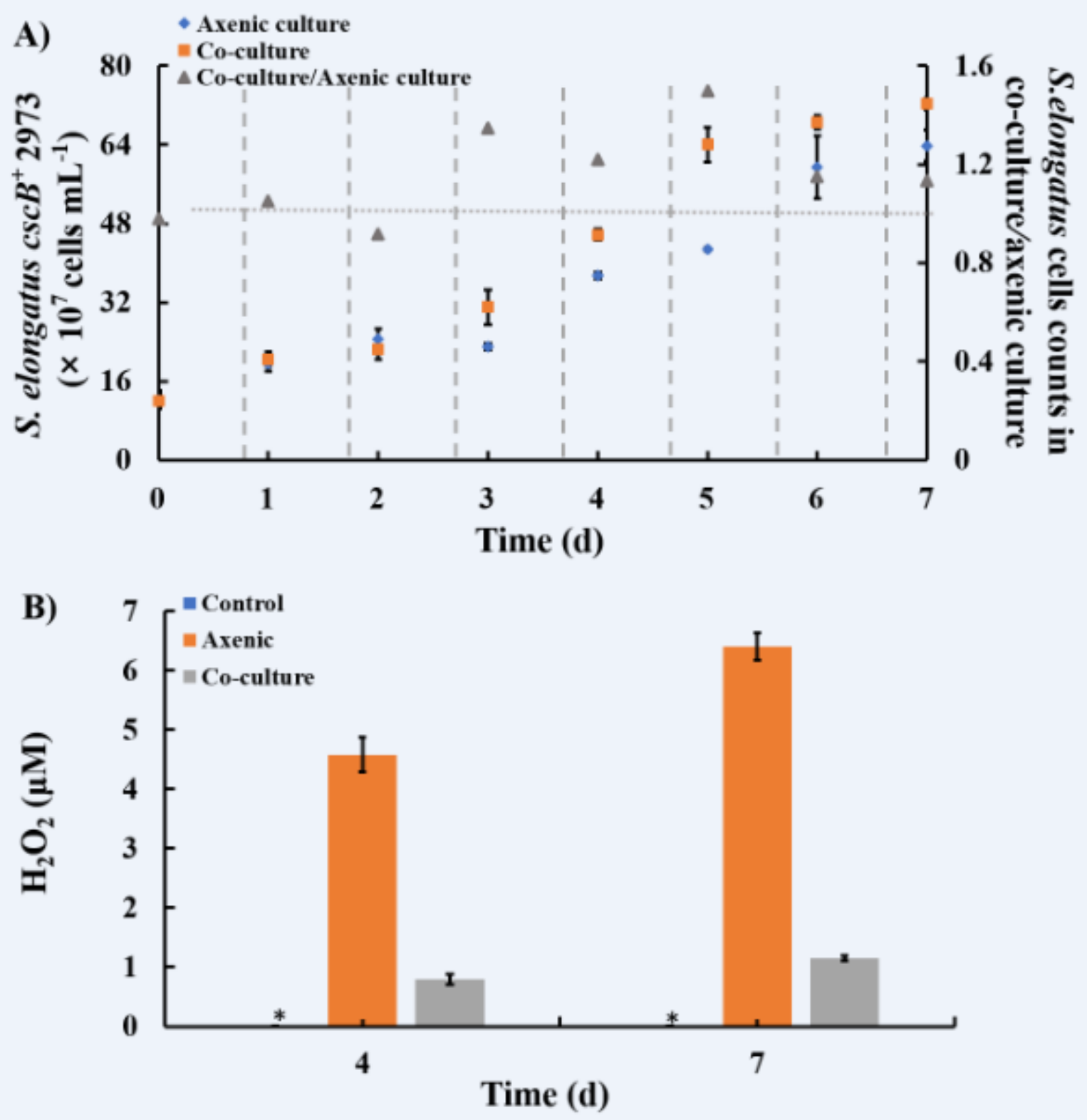

Figure 6

Growth of cyanobacteria and $\mathrm{H} 2 \mathrm{O} 2$ content in different culture conditions. (A) Difference in the growth of $\mathrm{S}$. elongatus $\mathrm{cscB}+$ in the artificial consortium and under axenic conditions. The blue rhombus represents the cell number in the axenic consortium, the orange rectangle represents the cell number in the artificial consortium, and the gray triangle represents the ratio between the two conditions. (B) $\mathrm{H} 2 \mathrm{O} 2$ content in blank CoBG-11 (control), axenic culture and co-culture systems under the same conditions $\left(30^{\circ} \mathrm{C}, 100 \mu \mathrm{E}\right.$ m-2s-1 light, $150 \mathrm{mM} \mathrm{NaCl}$; "*” indicates that no detectable amount was observed under the conditions). The error bars represent the calculated standard deviation of the measurements of three biological replicates. 


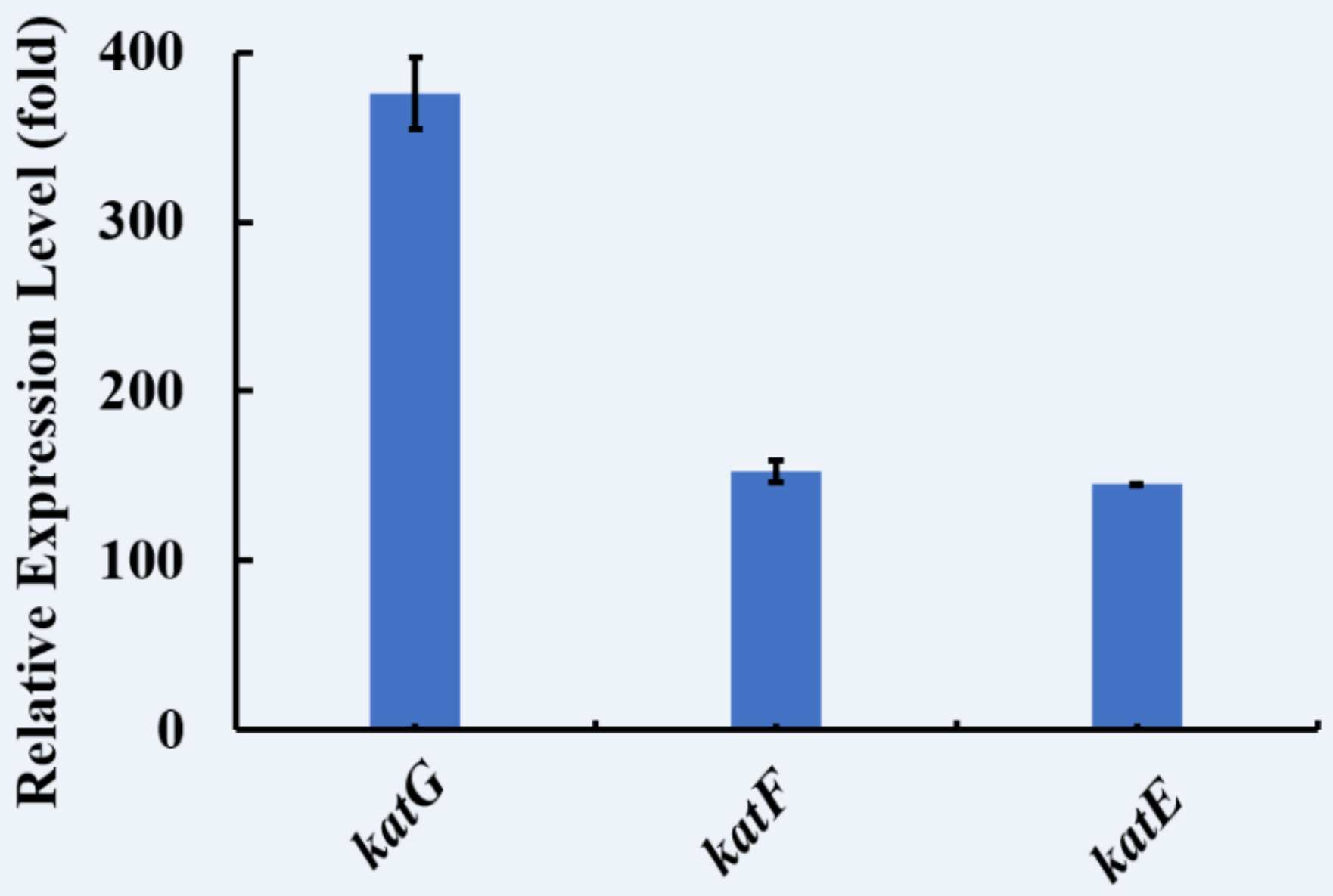

Figure 7

Expression level analysis of genes involved in ROS quenching. Gene expression analysis of katG, katF and katE in the ABKm strain.

\section{Supplementary Files}

This is a list of supplementary files associated with this preprint. Click to download.

- TableS1.docx

- Figs6.tif

- FigS5.tif

- Figs1.tif

- Figs4.tif

- FigS2.tif

- FigS3.tif 